\title{
Ordering Gold Nanoparticles with DNA Origami
} Nanoflowers

Robert Schreiber ${ }^{1, *}$, Ibon Santiago $^{1}$, Arzhang Ardavan ${ }^{1}$, Andrew J. Turberfield ${ }^{1, *}$

${ }^{1}$ University of Oxford, Department of Physics, Clarendon Laboratory, Parks Road, Oxford OX1 3PU, United Kingdom

*a.turberfield@physics.ox.ac.uk

ONE-SENTENCE SUMMARY: DNA origami nanoflowers control the bonding symmetry of gold nanoparticles, allowing the formation of ordered lattices.

KEY WORDS: DNA nanotechnology, nanostructure, origami, nanoparticle, selfassembly 
ABSTRACT Nanostructured materials, including plasmonic metamaterials made from gold and silver nanoparticles, provide access to new materials properties. The assembly of nanoparticles into extended arrays can be controlled through surface functionalization and the use of increasingly sophisticated linkers. We present a versatile way to control the bonding symmetry of gold nanoparticles by wrapping them in flower-shaped DNA origami structures. These 'nanoflowers' assemble into two-dimensonal gold nanoparticle lattices with symmetries that can be controlled through auxiliary DNA linker strands. Nanoflower lattices are true composites: interactions between the gold nanoparticles are mediated entirely by DNA, and the DNA origami will only fold into its designed form in the presence of the gold nanoparticles.

The directed assembly of composites incorporating metal nanostructures is an important route to materials with new physical properties, including plasmonic metamaterials. ${ }^{1}$ Previous work has shown that gold nanoparticles (NPs) can assemble to create nanoparticle molecules, clusters and crystals with different symmetries and interparticle spacings, ${ }^{2-5}$ and a new generation of asymmetric $\mathrm{NPs}^{6-10}$ promise to allow even more complex structures. Surface functionalization with single-stranded DNA (ssDNA) provides flexible control of interactions between metallic NPs, e.g., by preventing aggregation, ${ }^{3,411}$ reducing polydispersity ${ }^{4,12}$ and controlling valency. ${ }^{6}$ A key advantage of using ssDNA as a ligand is the possibility of engineering inter-particle interactions through sequence-specific hybridization of complementary linker strands..$^{2-5}$ Use of isotropic DNA coatings to provide short-range entropic repulsion and longer-range bonding interactions allows the formation of colloidal crystals: ${ }^{3-5}$ crystal symmetry and 
lattice spacing are controllable through linker lengths, particle sizes and patterns of pairwise bonding interactions. However, to generate arbitrary lattices and more complex metallic nanostructures, additional control of the geometry of inter-particle interactions through anisotropic functionalization ${ }^{13}$ is highly desirable. This can be achieved by attaching DNA-functionalized NPs to more complex DNA nanostructures ${ }^{9,10,14-18}$ capable of site-specific functionalization with nanometre precision. The DNA 'origami' technique, by which a (typically) 7-kbase DNA scaffold strand is folded into shape by hybridization to hundreds of synthetic 'staple' oligonucleotides, allows a very wide variety of templating structures to be formed. ${ }^{19-23}$ Here, we demonstrate the use of a hybrid gold-DNA origami nanostructure to provide flexible control of the formation of periodic arrays of AuNPs.

\section{Results/Discussion}

We designed a flower-like DNA origami structure, with a DNA-functionalized AuNP at the centre (Figure 1, Supporting Fig. S1), to be formed by annealing all components in a single assembly reaction (Figure 1a). The AuNP is surrounded by radial petals, approx. $20 \mathrm{~nm}$ in length, formed by bundles of four DNA helices (Figure 1b-d). The scaffold forms one strand of each helix, so each has a unique base sequence and can be specifically functionalized. Petals are linked at the centre of the structure by scaffold and staple crossovers (Figure 1d, Supporting Fig. S1). The four helices of each petal are tightly bound to each other by staple crossovers. There are 32 petals, alternately arranged in two layers of 16 (Figure 1b). The petals meet in the centre to form a hole of approx. $15 \mathrm{~nm}$ diameter, around which the DNA helices are stacked close together. On the inner end of each petal, two staples are extended to provide $\mathrm{A}_{n}(n=4-8)$ ssDNA 
overhangs positioned at the edge of the hole. These hybridize to multiple $\mathrm{T}_{19}$ oligonucleotides that are covalently attached to the surface of the $15 \mathrm{~nm}$ AuNP through 5' thiol modifications (Figure 1b), binding the AuNP in the centre of the nanoflower.

The nanoflower is created by annealing the DNA scaffold strand, the staple strands and the DNA-functionalized AuNPs (Method M1) from $65{ }^{\circ} \mathrm{C}$ to $20{ }^{\circ} \mathrm{C}$ over $16 \mathrm{hrs}$ (Method M2). Equal concentrations of AuNPs and origami scaffolds are used; staples are in $20 \times$ excess. The assembled hybrid structures are separated from excess staples and unbound AuNPs by gel electrophoresis (Figure 1e, Method M3). To achieve higher purity and concentration, the nanoflowers are pelleted by centrifugation after gel extraction and resuspended (Supporting Figure S2). Transmission electron micrographs (Figure 1f) show uniform, well-formed nanoflowers. The fraction of nanoflowers that are well-formed and contain a AuNP is approximately $80 \%$ after gel purification and approximately $99.5 \%$. after subsequent spin purification.

Annealing enables formation of the most stable nanostructure, in which the DNA origami petals are bound around the equator of a single AuNP. The central AuNP is an intrinsic part of the hybrid gold-DNA structure. If it is not present, the DNA petals do not unfold but stack approximately parallel to each other to form a rectangular block (Supporting Figure S3). Later addition of AuNPs does not produce significant conversion to nanoflowers, even after incubation at $25^{\circ} \mathrm{C}$ for $24 \mathrm{hrs}$.

Each part of the DNA origami is identifiable through its unique DNA base sequence, allowing selective functionalization of the outer ends of some petals with ssDNA linkers to allow control of subsequent lattice formation (Figure 2a). To create the lattices shown in Figure 2b-d we separately assembled two batches of nanoflowers, each with 
one of two complementary linking sequences (A or B type) repeated at two or four sites (Figure 2b, 2c-d respectively). Each attachment site comprises four petals, each carrying two ssDNA linkers (Figure 2a). Each linker consists of a spacer sequence of 10 bases (ACATCCAACCCC) to give flexibility and a hybridization sequence of 6 bases (TACTTC and GAAGTA for A and B, respectively). ${ }^{14}$ Lattices were assembled by mixing nanoflowers $\mathrm{A}$ and $\mathrm{B}$ in equal concentrations and annealing from $44{ }^{\circ} \mathrm{C}$ to $20{ }^{\circ} \mathrm{C}$ over 24 hrs. Hybridization between A and B linkers (Figure 2a) links the nanoflowers into lattices whose structures are controlled by the relative positions of the functionalized petals.

Figure 2 b-d shows three different lattices, corresponding to three different choices of functionalization sites. Two attachment sites separated by $180^{\circ}$ produce a chain of NPs (Figure 2b). Four attachment sites separated by $90^{\circ}$ produce a square NP lattice (Figure 2c). Hexagonal NP lattices were achieved by using four attachment sites separated by approximately $120^{\circ}$ and $60^{\circ}$ (at petal positions $1-4,7-10,18-21$, and $24-$ 27) (Figure 2d). (N.B. if distinction is made between NPs on the basis of the slight difference between A- and B-type linkers, then the corresponding primitive lattices are square and approximately rectangular, with primitive unit cells containing one of each NP type.) Supporting Fig S4 contains additional TEM images. Supporting Fig S5 shows nearest-neighbour distribution functions: an average inter-particle spacing of $62( \pm 4) \mathrm{nm}$ was measured for all lattices, consistent with the designed dimensions of the nanostructure. When lattices are formed at lower temperatures $\left(<30^{\circ} \mathrm{C}\right)$, the nanoflower petals can interpenetrate, decreasing by approximately $25 \%$ the inter-particle distance (Supporting Fig. S6). 
The AuNP size, and the distance between the gold surfaces, can be controlled by electroless deposition of gold ions from solution. ${ }^{24,25}$ Figure 3 shows the result of gold enhancement performed on the assembled one-dimensional chain after deposition on an electron microscopy grid (Method M4, Supporting Fig. S7). The reaction was stopped after $5 \mathrm{sec}$, , sufficient for AuNPs to grow from $15 \mathrm{~nm}$ to $60 \mathrm{~nm}$ diameter. The enlarged AuNPs just contact each other: the inter-particle spacing did not change measurably.

\section{Conclusions}

We have introduced origami nanoflowers, a class of composite gold - DNA nanostructure with flexible origami petals wrapped around single AuNPs. These are true composite structures: a nanoflower will not unfold unless it has a AuNP at the centre. The origami structures give control of the bonding valency and geometry for otherwise symmetric NPs, allowing facile control of lattice symmetry. Further control of particle size and spacing through electroless gold deposition after assembly could be used to control photonic properties: for example, self-assembled chains with tunable particle size could be used as plasmonic wave guides. ${ }^{26}$ Three-dimensional nanoflowers with radial petals can be envisaged, allowing the construction of three-dimensional NP crystals. Directional control of the assembly of NPs is an essential tool for the further exploration of functional nanostructures such as optical metamaterials ${ }^{27,28}$ and catalytic superlattices. $^{29}$ 


\section{Methods/Experimental}

\section{M1 | DNA functionalization of AuNPs.}

DNA functionalization mainly follows protocols from Ding et al. ${ }^{30}$ and Kuzyk et al. ${ }^{25}$ Citrate-stabilized colloidal $15 \mathrm{~nm}$ AuNPs (British Biocell International) were mixed with 2.5mM BSPP (Bis( $p$-sulfonatophenyl)phenylphosphine dihydrate dipotassium salt, Sigma-Aldrich) and incubated overnight. AuNPs were precipitated by addition of $\sim 0.2 \mathrm{M} \mathrm{NaCl}$ (until colour change occurred) and pelleted by centrifugation at 1,600g for $30 \mathrm{~min}$. AuNPs were resuspended in $2.5 \mathrm{mM}$ BSPP solution to a concentration of $200 \mathrm{nM}$ and mixed with a $1,000 \times$ excess of $5^{\prime}$-thiolated ssDNA (sequence $\mathrm{T}_{19}$, biomers.net) and $5 \mathrm{mM}$ TCEP (Tris(carboxyethyl)phosphine hydrochloride, SigmaAldrich). After continuous salt titration with $\mathrm{NaCl}$ up to $700 \mathrm{mM}$ overnight, the DNA functionalized AuNPs were purified from the excess of DNA using $100 \mathrm{kDa}$ spin filters (Amicon Ultra from Millipore).

\section{M2 | One-pot annealing of DNA origami structure with equimolar ratio of AuNPs.}

$10 \mathrm{nM}$ of P7560 DNA scaffold, ${ }^{19}$ a staple mix containing $200 \mathrm{nM}$ of each DNA staple, and $10 \mathrm{nM}$ of DNA-functionalized AuNPs were mixed in a buffer containing $10 \mathrm{mM}$ Tris, $1 \mathrm{mM}$ EDTA and $14 \mathrm{mM} \mathrm{MgCl} 2, \mathrm{pH} 8.0$ and annealed from $65^{\circ} \mathrm{C}$ to $25^{\circ} \mathrm{C}$ over 16 hours (linear temperature ramps: $65^{\circ} \mathrm{C}$ to $60^{\circ} \mathrm{C}, 0.1^{\circ} \mathrm{C} \min ^{-1}, 60^{\circ} \mathrm{C}$ to $40^{\circ} \mathrm{C}, 0.025^{\circ} \mathrm{C}$ $\min ^{-1} ; 40^{\circ} \mathrm{C}$ to $\left.25^{\circ} \mathrm{C}, 0.1^{\circ} \mathrm{C} \min ^{-1}\right)$.

\section{M3 | Gel purification and concentration of origami nanoflowers.}

A- and B-type nanoflowers were prepared separately. Assembled nanoflowers were separated from excess staples and unincorporated AuNPs by gel electrophoresis (Buffer 
A: $1.5 \%$ agarose; $45 \mathrm{mM}$ Tris-borate, $11 \mathrm{mM} \mathrm{MgCl}_{2}, 1 \mathrm{mM}$ EDTA, $\mathrm{pH} 8.3 ; 8^{\circ} \mathrm{C} ; 4 \mathrm{hrs}$ at $3.5 \mathrm{Vcm}^{-1}$ ) (Figure 1e). The nanoflower band was cut from the gel and further diced; nanoflowers were extracted by squeezing the gel pieces between Parafilm ${ }^{\circledR}$-covered surfaces. The resulting droplet was recovered with a pipette and topped up to $1 \mathrm{~mL}$ of Buffer A. The extracted nanoflowers were centrifuged $\left(5,000 \mathrm{~g}\right.$ at $4^{\circ} \mathrm{C}$ for $30 \mathrm{~min}$.) to remove residual gel fragments and the supernatant was kept for the next step (Supporting Figure S2a). Nanoflowers were then pelleted by centrifugation at 8,000 g at $4{ }^{\circ} \mathrm{C}$ for $30 \mathrm{~min}$ to separate them from origami structures without AuNPs $(<25 \%)$ which were discarded in the supernatant (Supporting Figure S2b). Pelleted nanoflowers were resuspended in $\sim 10 \mu \mathrm{L}$ of Buffer A to a concentration of approx. $25 \mathrm{nM}$ (measured by the absorption spectrum of the AuNPs). A- and B-type nanoflowers were then mixed together in an equimolar ratio. Nanoflowers were pelleted again $\left(5,000 \mathrm{~g}\right.$ at $4{ }^{\circ} \mathrm{C}$ for $\left.15 \mathrm{~min}\right)$ to achieve higher concentrations $(>25 \mathrm{nM})$ needed for the creation of the lattices. The nanoflower pellet was topped up to $20 \mu \mathrm{L}$ of Buffer A and annealed from $44{ }^{\circ} \mathrm{C}$ to $20^{\circ} \mathrm{C}$ over $24 \mathrm{hrs}$ (linear temperature ramps: $44^{\circ} \mathrm{C}$ to

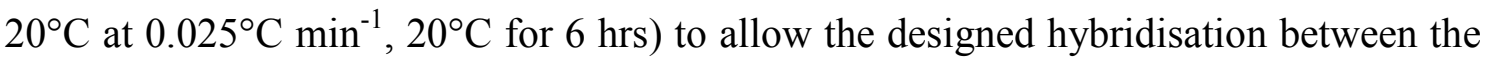
nanoflower types (A and B) to occur.

\section{M4 | Electroless deposition of metal ions onto assembled nanoflower chains.}

$10 \mu \mathrm{L}$ of $5 \mathrm{nM}$ nanoflowers, assembled into chains, was applied to a carbon-formvar copper TEM grid for $30 \mathrm{sec}$. Excess sample was removed with filter paper and the grid allowed to dry. $10 \mu \mathrm{L}$ of EM GoldEnhance ${ }^{\mathrm{TM}}$ (Nanoprobes) was applied to the dried nanoflower chains. After $5 \mathrm{sec}$ the enhancement was stopped by rinsing with $10 \mu \mathrm{L}$ $\mathrm{H}_{2} \mathrm{O}$. Nanoflowers were stained with uranyl acetate ${ }^{31}$ and imaged by transmission electron microscopy (FEI Tecnai 12 at 120kV). 
Conflict of Interest: The authors declare no competing financial interest.

Acknowledgement: This work was funded by the Oxford Martin School Programme on Bio-Inspired Quantum Technologies, the Department of Education and Culture of the Basque Government and a Royal Society-Wolfson Research Merit Award (AJT).

Supporting Information Available: Supporting Figures and Table. The Supporting Information is available free of charge on the ACS Publications website http://pubs.acs.org/. 


\section{References}

1. Soukoulis, C.; Wegener, M., Past Achievements and Future Challenges in the Development of Three-Dimensional Photonic Metamaterials. Nat. Photonics 2011, 5, 523-530.

2. Alivisatos, A.; Johnsson, K.; Peng, X.; Wilson, T.; Loweth, C.; Bruchez, M.; Schultz, P., Organization of 'Nanocrystal Molecules' Using DNA. Nature 1996, 382, 609-611.

3. Nykypanchuk, D.; Maye, M.; van der Lelie, D.; Gang, O., DNA-Guided Crystallization of Colloidal Nanoparticles. Nature 2008, 451, 549-552.

4. Mirkin, C.; Letsinger, R.; Mucic, R.; Storhoff, J., A DNA-Based Method for Rationally Assembling Nanoparticles into Macroscopic Materials. Nature 1996, 382, 607-609.

5. Kim, Y.; Macfarlane, R.; Jones, M.; Mirkin, C., Transmutable Nanoparticles with Reconfigurable Surface Ligands. Science 2016, 351, 579-582.

6. Edwardson, T.; Lau, K.; Bousmail, D.; Serpell, C.; Sleiman, H., Transfer of Molecular Recognition Information from DNA Nanostructures to Gold Nanoparticles. Nat. Chem. 2016, 8, 162-170.

7. Sun, W.; Boulais, E.; Hakobyan, Y.; Wang, W.; Guan, A.; Bathe, M.; Yin, P., Casting Inorganic Structures with DNA Molds. Science 2014, 346, 12583611258361.

8. Xu, X.; Rosi, N.; Wang, Y.; Huo, F.; Mirkin, C., Asymmetric Functionalization of Gold Nanoparticles with Oligonucleotides. J. Am. Chem. Soc. 2006, 128, 9286-9287.

9. Li, Y.; Liu, Z.; Yu, G.; Jiang, W.; Mao, C., Self-Assembly of Molecule-like Nanoparticle Clusters Directed by DNA Nanocages. J. Am. Chem. Soc. 2015, $137,4320-4323$.

10. Zhao, Z.; Jacovetty, E.; Liu, Y.; Yan, H., Encapsulation of Gold Nanoparticles in a DNA Origami Cage. Angew. Chem., Int. Ed. 2011, 50, 2041-2044.

11. Sato, K.; Hosokawa, K.; Maeda, M., Rapid Aggregation of Gold Nanoparticles Induced by Non-Cross-Linking DNA Hybridization. J. Am. Chem. Soc. 2003, $125,8102-8103$.

12. Storhofff, J.; Elghanian, R.; Mirkin, C.; Letsinger, R., Sequence-Dependent Stability of DNA-Modified Gold Nanoparticles. Langmuir 2002, 18, 6666-6670.

13. Wang, Y.; Wang, Y.; Breed, D.; Manoharan, V.; Feng, L.; Hollingsworth, A.; Weck, M.; Pine, D., Colloids with Valence and Specific Directional Bonding. Nature 2012, 491, 51-55. 
14. Tian, Y.; Wang, T.; Liu, W.; Xin, H.; Li, H.; Ke, Y.; Shih, W.; Gang, O., Prescribed Nanoparticle Cluster Architectures and Low-Dimensional Arrays Built Using Octahedral DNA Origami Frames. Nat. Nanotechnol. 2015, 10, 637644.

15. Zheng, J.; Constantinou, P.; Micheel, C.; Alivisatos, A.; Kiehl, R.; Seeman, N., Two-Dimensional Nanoparticle Arrays Show the Organizational Power of Robust DNA Motifs. Nano Lett. 2006, 6, 1502-1504.

16. Ke, Y.; Ong, L.; Sun, W.; Song, J.; Dong, M.; Shih, W.; Yin, P., DNA Brick Crystals with Prescribed Depths. Nat. Chem. 2014, 6, 994-1002.

17. Tian, Y.; Zhang, Y.; Wang, T.; Xin, H.; Li, H.; Gang, O., Lattice Engineering Through Nanoparticle-DNA Frameworks. Nat. Mater. 2016, 15, 654-661.

18. Liu, W.; Tagawa, M.; Xin, H.; Wang, T.; Emamy, H.; Li, H.; Yager, K.; Starr, F.; Tkachenko, A.; Gang, O., Diamond Family of Nanoparticle Superlattices. Science 2016, 351, 582-586.

19. Rothemund, P., Folding DNA to Create Nanoscale Shapes and Patterns. Nature 2006, 440, 297-302.

20. Douglas, S.; Dietz, H.; Liedl, T.; Hogberg, B.; Graf, F.; Shih, W., Self-Assembly of DNA into Nanoscale Three-Dimensional Shapes. Nature 2009, 459, 414-418.

21. Dietz, H.; Douglas, S.; Shih, W., Folding DNA into Twisted and Curved Nanoscale Shapes. Science 2009, 325, 725-730.

22. Liedl, T.; Hogberg, B.; Tytell, J.; Ingber, D.; Shih, W., Self-Assembly of ThreeDimensional Prestressed Tensegrity Structures from DNA. Nat. Nanotechnol. 2010, 5, 520-524.

23. Benson, E.; Mohammed, A.; Gardell, J.; Masich, S.; Czeizler, E.; Orponen, P.; Hogberg, B., DNA Rendering of Polyhedral Meshes at the Nanoscale. Nature 2015, 523, 441-444.

24. Pilo-Pais, M.; Goldberg, S.; Samano, E.; LaBean, T.; Finkelstein, G., Connecting the Nanodots: Programmable Nanofabrication of Fused Metal Shapes on DNA Templates. Nano Lett. 2011, 11, 3489-3492.

25. Kuzyk, A.; Schreiber, R.; Fan, Z.; Pardatscher, G.; Roller, E.; Hogele, A.; Simmel, F.; Govorov, A.; Liedl, T., DNA-Based Self-Assembly of Chiral Plasmonic Nanostructures with Tailored Optical Response. Nature 2012, 483, 311-314.

26. Maier, S.; Kik, P.; Atwater, H.; Meltzer, S.; Harel, E.; Koel, B.; Requicha, A., Local Detection of Electromagnetic Energy Transport Below the Diffraction Limit in Metal Nanoparticle Plasmon Waveguides. Nat. Mater. 2003, 2, 229-232. 
27. Schreiber, R.; Luong, N.; Fan, Z.; Kuzyk, A.; Nickels, P.; Zhang, T.; Smith, D.; Yurke, B.; Kuang, W.; Govorov, A.; Liedl, T., Chiral Plasmonic DNA Nanostructures with Switchable Circular Dichroism. Nat. Commun. 2013, 4.

28. Pendry, J., Negative Refraction Makes a Perfect Lens. Phys. Rev. Lett. 2000, 85, 3966-3969.

29. Auyeung, E.; Morris, W.; Mondloch, J.; Hupp, J.; Farha, O.; Mirkin, C., Controlling Structure and Porosity in Catalytic Nanoparticle Superlattices with DNA. J. Am. Chem. Soc. 2015, 137, 1658-1662.

30. Ding, B.; Deng, Z.; Yan, H.; Cabrini, S.; Zuckermann, R.; Bokor, J., Gold Nanoparticle Self-Similar Chain Structure Organized by DNA Origami. J. Am. Chem. Soc 2010, 132, 3248-3249.

31. Douglas, S.; Chou, J.; Shih, W., DNA-Nanotube-Induced Alignment of Membrane Proteins for NMR Structure Determination. Proc. Natl. Acad. Sci. U. S. A. 2007, 104, 6644-6648. 


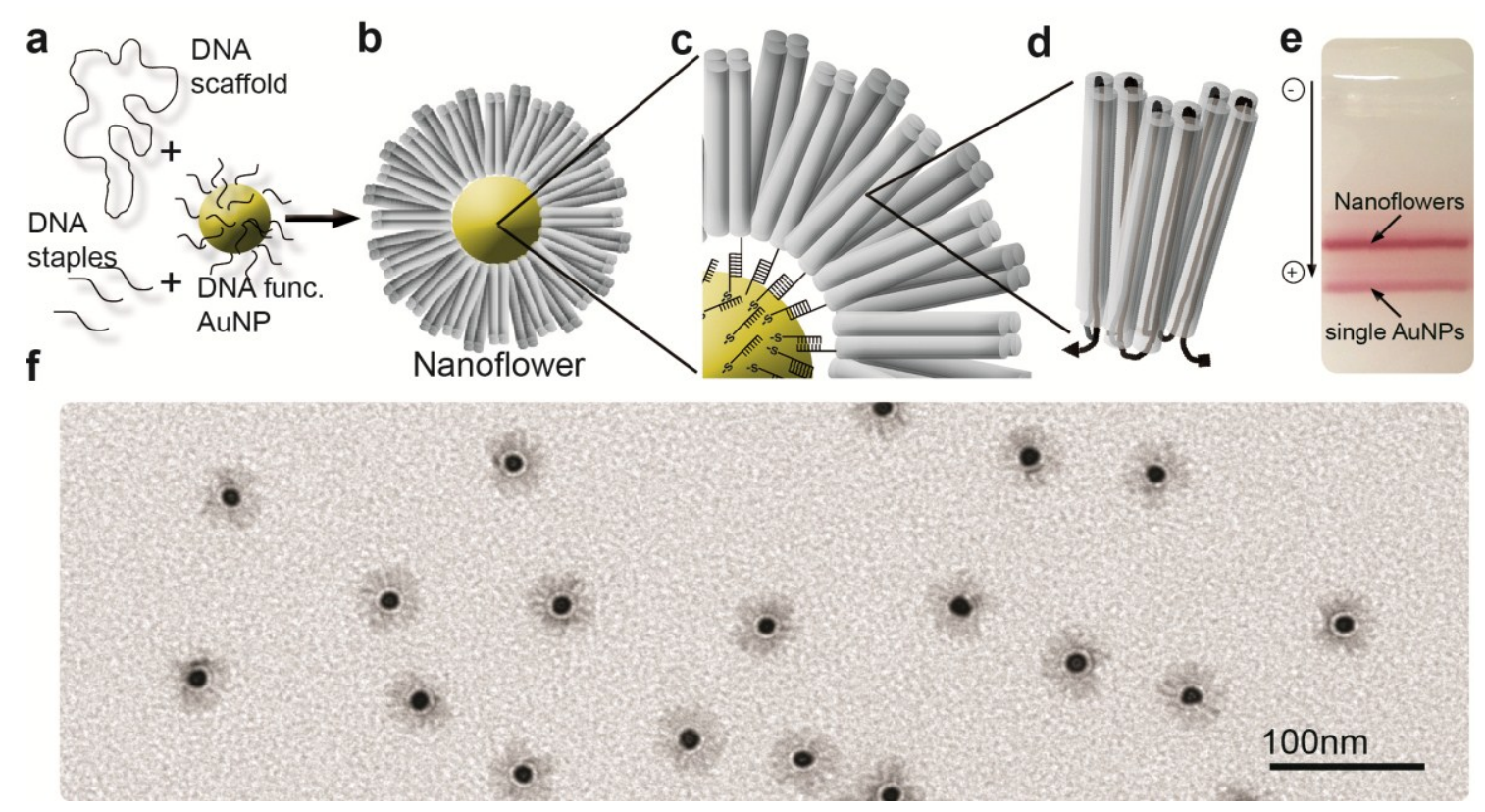

Figure 1 | DNA origami nanoflower. a, Nanoflower assembly: ssDNA scaffold (7249 nucleotides) from bacteriophage M13 is annealed with 244 synthetic 'staple' oligonucleotides (typ. 40 bases) and $15 \mathrm{~nm}$ AuNPs, functionalized with multiple covalently-bound oligonucleotides, in a one-pot reaction. b-d, Nanoflower structure. A DNA-functionalized AuNP is wrapped by a single DNA origami comprising 32 radially arranged petals, each consisting of four cross-linked DNA helices. Oligonucleotides attached to the AuNP hybridize to complementary ssDNA extensions to staples in the centre of the origami to bind the AuNP in the centre of the nanoflower. The scaffold strand (black) runs through each helix of the nanoflower petals. e, Annealed nanoflowers were purified from the excess of staples and unbound AuNPs by agarose gel electrophoresis. Nanoflowers were separated on a $1.5 \%$ gel for $4 \mathrm{hrs}$ at $3.5 \mathrm{Vcm}^{-1}$. The thick red band contains the nanoflowers. The faster, dimmer band contains unincorporated AuNPs. Slower bands contain nanoflower dimers and aggregates. f, Transmission electron micrograph (stained with uranyl acetate) showing AuNPs in the centre of each nanoflower. 
a

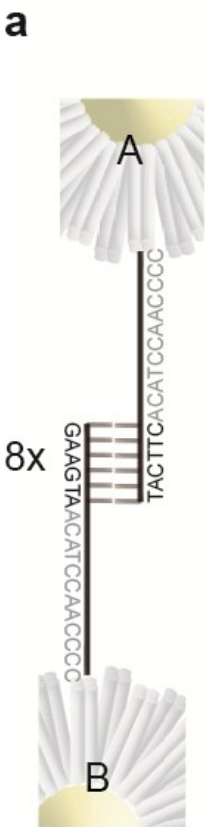

b
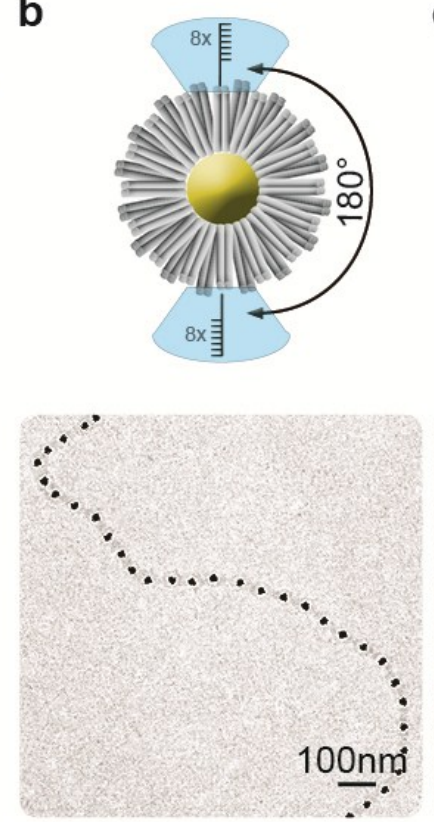

C
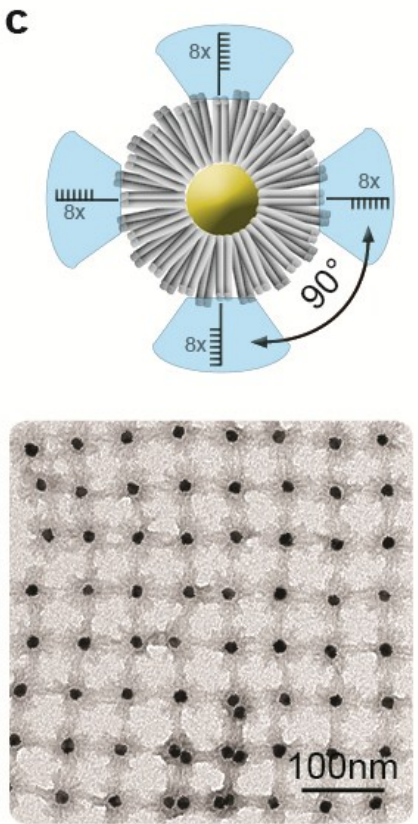

d
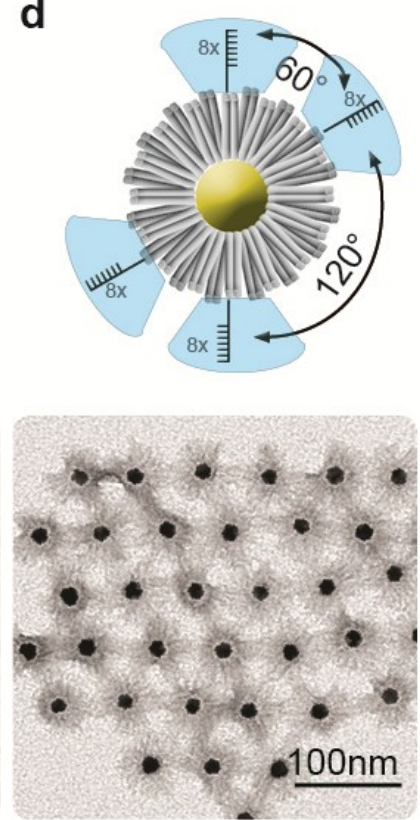

Figure 2 | Ordering AuNPs into different lattices by changing the valency of the nanoflowers. a, Hybridization scheme (not to scale): A- and B-type nanoflowers are linked by hybridization of complementary linker oligonucleotides ( 8 handles per attachment site). b, Chains of AuNPs were obtained by providing A and B nanoflowers with two hybridization sites separated by $180^{\circ}$. c, Square lattice obtained with four hybridization sites at $90^{\circ}$. d, Hexagonal lattices obtained with four hybridization sites with approx. $60^{\circ}$ and $120^{\circ}$ separation. (See Supporting Fig. S4 for additional electron micrographs.) 

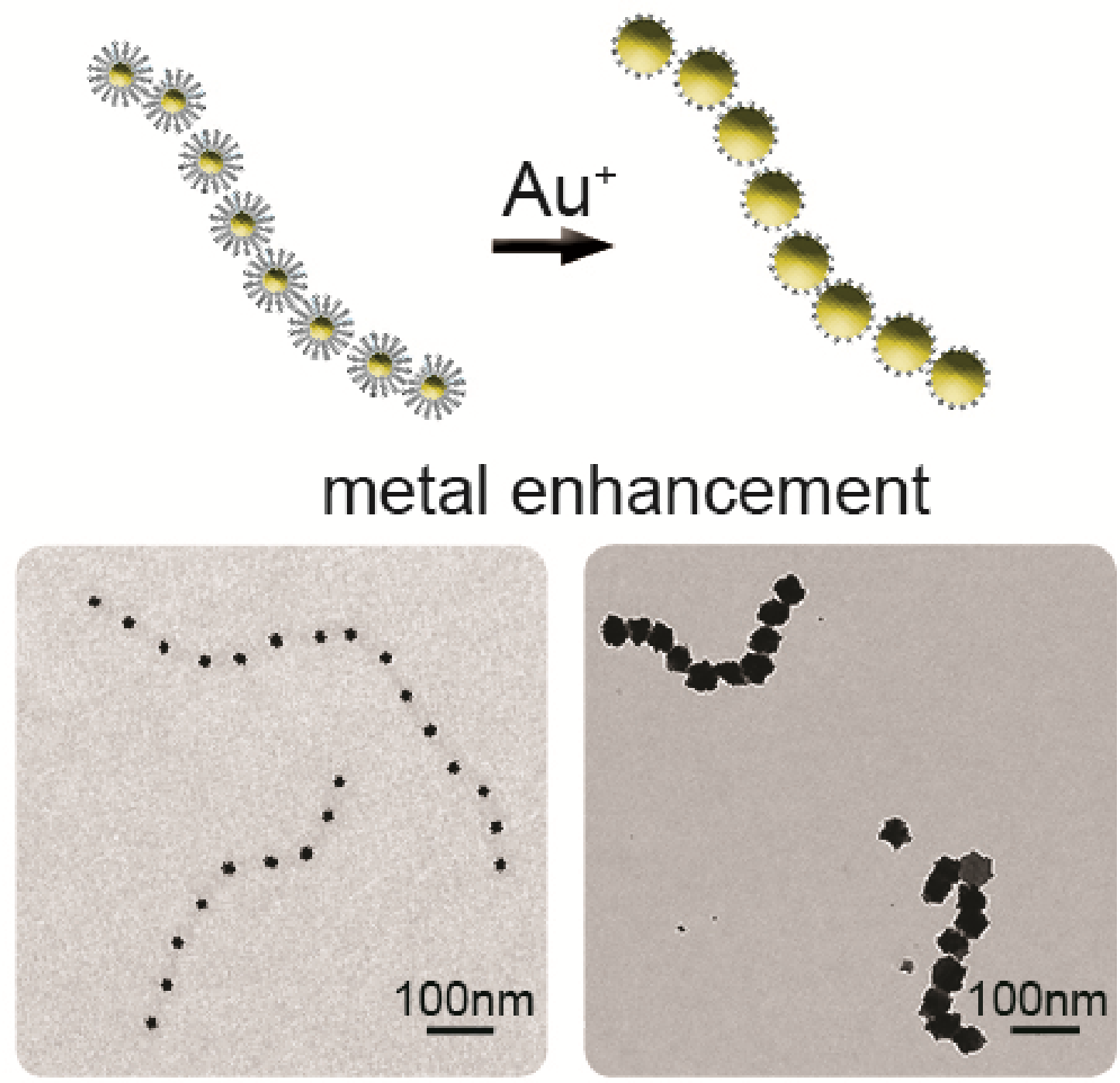

Figure 3 | Gold enhancement of nanoflower chains. The diameter of the gold nanoparticles was increased from $15 \mathrm{~nm}$ to $\sim 60 \mathrm{~nm}$, after assembly of the chain, by electroless deposition. The particle spacing remained approximately constant at $60 \mathrm{~nm}$. 